\title{
Parallel discrimination of subjective contours defined by offset gratings
}

\author{
RICK GURNSEY \\ Concordia University, Montreal, Quebec, Canada \\ and \\ G. KEITH HUMPHREY and PAULA KAPITAN \\ University of Western Ontario, London, Ontario, Canada
}

\begin{abstract}
Recent physiological studies (von der Heydt \& Peterhans, 1989) suggest that the orientation of subjective contours is encoded very early in the visual system (V2 in monkey). This result is seemingly at odds with existing psychophysical data which suggest that the detection of subjective contours involves selective attention. It is argued that certain subjective contours are registered in a reflexive (bottom-up) manner by the visual system but that selective attention may be needed to gain access to this representation. To assess this suggestion, a visual-search task was used in which subjects were to detect the presence of a horizontal (vertical) subjective contour (defined by offset gratings) in a variable number of vertical (horizontal) subjective contours (also defined by offset gratings). When there were no competing organizations within the display, detection was indeed independent of the number of nontarget distractors, that is, selective attention was unnecessary. In a second experiment, we found that a curved form (a crescent defined by subjective contours) was easier to detect in a background of vertical bars (also defined by subjective contours) than vice versa, namely, a search asymmetry paralleling those found by Treisman and Gormican (1988). A final experiment showed that when the horizontal and vertical bars of the first experiment formed textured regions, they could be discriminated at very brief display durations (30-120 msec). However, when the line terminations aligned along the subjective contour were tapered rather than abrupt, discrimination dropped off with the degree of tapering. The latter result is consistent with the assumption that the registration of subjective contours in V2 involves the integration of responses from aligned, end-stopped cells found in V1 (von der Heydt \& Peterhans, 1989).
\end{abstract}

It is common to emphasize the importance of image contours because of their relationship to object boundaries and surface discontinuities in the scene (e.g., Marr, 1982). Often, object boundaries and surface discontinuities exist as luminance changes in the image, but this is not always the case. Although we frequently perceive clear perceptual boundaries between image regions, the physical bases for these percepts may be very slight. Figure 1, for example, shows two cases in which contours are perceived yet do not exist as luminance gradients. Such contours are generally referred to as subjective or gradient-free contours and have been of great interest to psychologists since Schumann's (1900/1987) original paper on the topic. In recent years attention has been increasingly devoted to subjective contours (e.g., Petry \& Meyer, 1987). The Kaniza-type subjective contour (Figure 1A) and offset grating contours (Figure 1B) are

This work was supported by NSERC grants to R. G. and G. K. H. Portions of this work were presented at the meeting of the Association for Research in Vision and Ophthalmology, 1990. Correspondence should be addressed to R. Gurnsey, Department of Psychology, Concordia University, 7141 Sherbrooke St. W., Montreal, PQ, Canada H4B IR6.
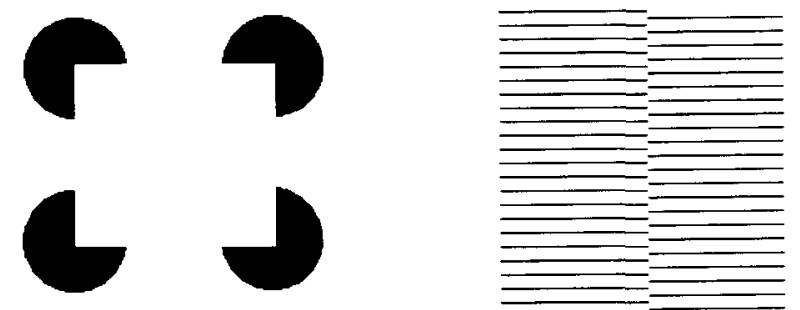

Figure 1. Two examples of subjective contours. In the left panel, an illusory contrast is seen between the interior of the square and the white background, although there is no luminance gradient present. In the right panel, a strong perceptual boundary is seen dividing the left and right halves of the display. Again, no luminance gradient exists.

among the most commonly studied subjective contours. A question often asked is whether the visual representations induced by such stimuli-as shown in Figure 1are computed in a bottom-up, reflexive manner or whether they are the result of more deliberate, inferential processes associated with selective attention.

Several results suggest that subjective contours are, indeed, coded in a reflexive manner at a very early stage of visual processing. For example, it has been shown that 
subjective contours (as in Figures 1A and 1B) can induce tilt aftereffects (Paradiso, Shimojo, \& Nakayama, 1989; Smith \& Over, 1975) and motion aftereffects (Smith \& Over, 1979). Such aftereffects are generally associated with adaptation of low-level, spatiotemporal filters. The strongest support for this interpretation comes from the recent physiological research of von der Heydt, Peterhans, and Baumgartner (1984, Peterhans \& von der Heydt, 1989; von der Heydt \& Peterhans, 1989), who have found that many cells in V2 of monkey may be considered generalized contour-selective mechanisms in that they respond to both luminance and gradient-free contours of the same orientation. Such cells would respond to the orientation of the subjective contour in Figure 1B and not to the orientation of the grating lines. It is important to note that recordings were made while the monkey attended stimuli outside the cells' receptive fields (von der Heydt et al., 1984), suggesting that attention is not necessary for the registration of such contours. Other physiological data implicating early neural mechanisms in the detection of subjective contours were provided by Redies (1989), who found that complex cells in cat respond to such contours. Both sorts of mechanisms may involve integration of the outputs of end-stopped, simple cells (e.g., Kato, Bishop, \& Orban, 1978). Adaptation of such neural organizations could be the neural correlate for the tilt aftereffects just mentioned (Paradiso et al., 1989).

The results just cited put the registration of subjective contours at the same level as the registration of luminance contours in terms of anatomical locus. There are, however, equally compelling reasons for adopting a top-down explanation. For example, certain subjective contours are often not perceived unless the subject's attention is drawn to them (Rock, 1987). Also, subjective contours may be ambiguous, permitting two possible organizations that are, to some extent, under the subject's control. If subjective contours are computed in a bottom-up, or reflexive, fashion, then they should exhibit the characteristics of preattentive processes (Neisser, 1967). Namely, their detectability should not be influenced by concurrent, nonvisual task requirements (Pritchard \& Warm, 1983), they should show spontaneous segregation in a texturediscrimination task (Beck, 1982; Julesz, 1984), and they should be detected in parallel in a visual-search task (Treisman \& Gelade, 1980). Recent evidence indicates that subjective contour perception demonstrates none of these characteristics (Grabowecky \& Treisman, 1989; Meyer \& Fish, 1987; Pritchard \& Warm, 1983).

\section{Dual Task Procedures}

Pritchard and Warm (1983) examined whether form discrimination based on Kaniza-like subjective contours versus real contours was differentially affected by attentional load. They found that without attentional load there was no significant difference in the speed with which same-different judgments could be made between forms defined by real or subjective contours. However, when a concurrent, attention-demanding (digit-span) task was added, for same trials at least, reaction times increased more for the subjective contours than for the real contours. The implication is that processing subjective contours is a resource-demanding operation that can be impaired by additional demands on resources.

\section{Texture Segregation}

Meyer and Fish (1987) showed that subjective contours do not elicit strong texture segregation. Their displays comprised 36 elements arranged in a $6 \times 6$ array, each element of which was composed of three "pacman" figures (see Figure 2). In a central $2 \times 2$ subarray, the pacmen were arranged to form subjective triangles, whereas the remaining pacmen were arranged in random orientations. The central region of subjective contours did not spontaneously segregate from the rest of the display. In fact, these displays were rated by subjects to be the lowest of all displays tested in eliciting strong texture segregation. Thus, subjective contours defined in this way do not provide the strong texture discrimination found with other forms (Beck, 1982; Gurnsey \& Browse, 1987, 1989; Julesz, 1984).

\section{Visual Search}

Finally, Grabowecky and Treisman (1989) used materials similar to those of Meyer and Fish (1987) in a visualsearch task. They asked subjects to detect the presence of a single subjective triangle amid distractors (constructed from the same pacmen) that did not create a subjective form (see Figure 2). They found that search time (i.e., time to detect the target) was strongly influenced by the number of background distractors. This suggests that attention had to be allocated to individual display locations to assess whether or not there was a subjective contour at that location, that is, detection required selective attention.

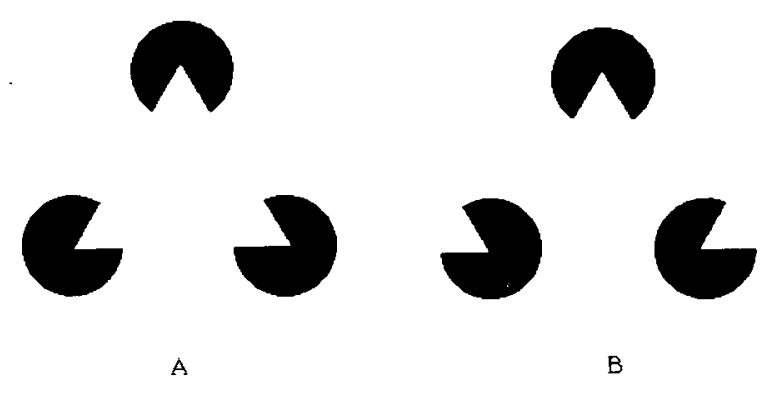

Figure 2. The left panel shows a subjective triangle defined by three pacmen, and the right panel shows an arrangement of similar pacmen that do not form a subjective figure. Meyer and Fish (1987) found that textures containing a central $2 \times 2$ region of elements similar to those in the left panel embedded in a larger background of 32 elements similar to those in the right panel (with the pacmen in random orientations) did not elicit strong segregation. Similarly, Grabowecky and Treisman (1989) found that the time required to detect a form such as that in the left panel in a variable number of distractors such as those in the right panel was strongly dependent on the number of background distractors. 
Thus, three results (Grabowecky and Treisman, 1989; Meyer \& Fish, 1987; Pritchard \& Warm, 1983) implicate attention in subjective contour processing. If selective attention is identified with deliberate, inferential processes, then these results are at odds with the physiological data (Redies, 1989; von der Heydt, et al., 1984). A possible reason for this discrepancy has to do with the stimuli used. The three experiments just described all utilized Kaniza-type figures with high-contrast pacmen as inducing stimuli. If it is assumed that subjective contours are treated as low-contrast luminance edges by the visual system, then it is reasonable to expect that the preponderance of high-contrast edges elsewhere in the display will impair detection of the subjective contours. For example, displays of the sort utilized by Meyer and Fish and Grabowecky and Treisman may be organized initially as a homogeneous field of dark blobs that obscure the subjective contours. This is essentially the explanation given by Meyer and Fish for the fact that subjective triangles did not strongly segment from the rest of the display. Consistent with this view are demonstrations by Fox (1978; Fox \& Mayhew, 1979) that effortless texture discrimination can be impaired by enclosing with a circle each texture micropattern in the disparate and background regions of the display. This introduces a level of homogeneity across the display that competes with differences that exist between subregions. Similar results have been reported by Caelli (1980).

Alternatively, if the subjective contours are to be treated as low-contrast luminance contours by the visual system, they will be in competition with the high-contrast luminance contours elsewhere in the display. Just as attention may be needed to gain access to the presence of a low-contrast luminance contour in the context of many high-contrast luminance contours, it may be needed to detect a subjective contour among many high-contrast luminance contours. Attention may then act to improve the signal-to-noise ratio only and would not be identified with resource-demanding, constructive processes applied selectively and sequentially to different display locations.

The physiological data suggest that the subjective contours are induced automatically by the presentation of the stimulus, but we suggest that access to that representation may be impaired by context. If it were possible to eliminate the competition provided by the high-contrast pacmen in the experiments just described, then according to our argument, the subjective contours should be detected in parallel in a visual-search task. Unfortunately, such a manipulation is impossible with the Kaniza-like (pacman-induced) stimuli since they act to induce the percept in the first place and reducing their contrast reduces the strength of the percept (Warm, Dember, Padich, Beckner, \& Jones, 1987).

Experiment $1 \mathrm{~A}$ addressed this issue by presenting subjective contours in a context intended to minimize extraneous masking influences. This was done by moving away from the Kaniza-type subjective contours and using subjective contours induced by offset lines (Paradiso et al., 1989; Vogels \& Orban, 1987). From our analysis, it would follow that access to subjective contours should be parallel when competing organizations are minimized.

\section{EXPERIMENT 1A}

Orientation discrimination is perhaps the simplest form discrimination that there is. As noted, recent psychophysical (Paradiso et al., 1989) and physiological (von der Heydt \& Peterhans, 1989) results suggest that the orientations of subjective contours are encoded very early in the visual process. It would be expected that a single vertical subjective contour should be detected in a background of horizontal subjective contours in time that is independent of the number of background distractors when competing stimuli are eliminated from the display. Constructing stimuli that permit an evaluation of this idea is a somewhat tricky task. From the earlier discussion, it can be appreciated that Kaniza-type subjective contours are inappropriate for such a question (cf. Grabowecky \& Treisman, 1989). Our solution to this issue and the logic of Experiment 1A can be seen in Figure 3.

To address the preattentive discrimination of subjective contours, we used a visual-search task (Treisman \& Gelade, 1980) in which subjects were to detect a single target element in a variable number of distractors as quickly and as accurately as possible. In Condition 1 (Figure 3, top panel), the targets and distractors were defined by bars of diagonally oriented black lines on a white background, constituting a low-resolution luminance gradient. This acts as a control condition to ensure that the orientation discrimination can in fact be performed in parallel. In Condition 2 (Figure 3, middle panel), the targets and distractors were subjective contours defined by displacing a bar of the diagonally oriented lines with respect to its background within a circular patch of diagonally

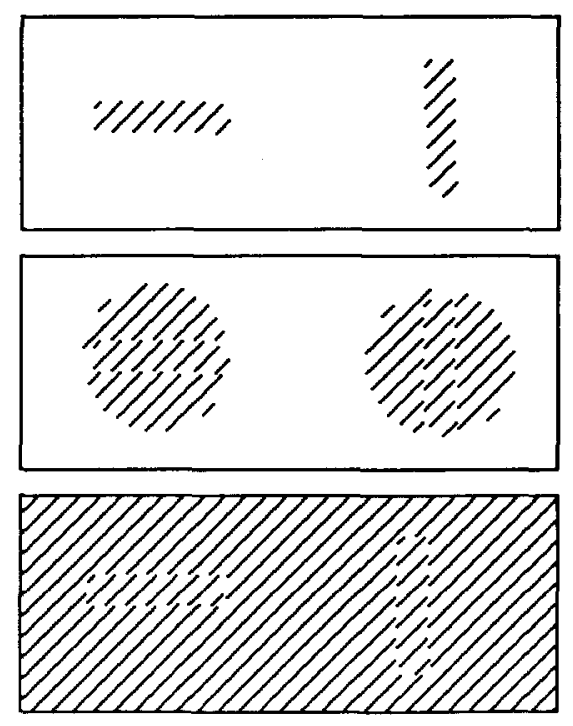

Figure 3. Examples of the stimuli used in the three conditions of Experiment 1A. See the text for an explanation. 
oriented lines. In this condition, the targets and distractors are intended to correspond to the materials used by Grabowecky and Treisman (1989). While the targets and distractors are distinguished only by the orientations of the subjective contours, search should be dependent on the number of background distractors because of the interfering influence of the circles in which they are enclosed. ${ }^{1}$ In Condition 3 (Figure 3, bottom panel), the targets and distractors were subjective contours defined by displacing a bar of grating with respect to its background, which was an entire field of diagonally oriented lines. In this condition, the posited masking influence of the disks in Condition 2 is eliminated.

To review, if orientation differences defined by subjective contours are detected in parallel, then Condition 3 should produce search rates that are independent of the number of background distractors. The same might be true of Condition 2 because the target and distractors differ with respect to the orientation of the subjective contours. However, it is expected that enclosing the subjective contours with disks will interfere with their detection and discrimination and therefore produce search rates that are dependent on the number of background distractors.

\section{Method}

Subjects. Thirty-six naive subjects were drawn from the undergraduate subject pool of the Psychology Department at the University of Western Ontario. All subjects reported normal or corrected-to-normal vision and received partial course credit for their participation.

Apparatus. All aspects of display presentation and response recording were under the control of an Apple Macintosh II computer using the VSearch package developed at the Vision Lab of the University of British Columbia (Enns, Ochs, \& Rensink, 1990). The display was a 13-in. Apple color monitor with a resolution of 640 $\times 480$ pixels.

Displays. The dimensions of the screen's display area were approximately $24 \mathrm{~cm}$ horizontal $\times 18 \mathrm{~cm}$ vertical. All displays involved $45^{\circ}$ gratings, which were high-contrast black lines (1 pixel wide) drawn on a white background and having a spatial period of 8 pixels. The horizontal and vertical rectangles used as stimuli were 12 pixels vertical (horizontal) by 50 pixels horizontal (vertical), which corresponded to bars $4 \times 18 \mathrm{~mm}$ on the screen. Displays were viewed from a distance of $50 \mathrm{~cm}$.

Procedure. Twelve subjects were assigned to each of the three conditions described above. On a single trial 1,6 , or 12 items were presented. Half the trials consisted of only nontargets, and on the other half a single target was presented among 0,5 , or 11 distractors. The positions of targets and distractors were randomized on each trial. Six of the subjects in each condition were asked to detect the presence of a vertical bar, and the remaining 6 were asked to detect the presence of a horizontal bar. Prior to the presentation of the test materials, the subjects were presented with a white field in Conditions 1 and 2 and with a homogeneous field of diagonal lines in Condition 3. The test materials were presented until the subjects made a response, that is, pressed one button to indicate that the target was present or another to indicate that the target was absent. After each trial a " +" or "-" appeared on the screen for $750 \mathrm{msec}$ to indicate if the response was correct or incorrect. Each trial was initiated by the computer $750 \mathrm{msec}$ after the offset of the feedback from the previous trial. The subjects were asked to respond as quickly and as accurately as possible.

Trials were presented in blocks of 48. Within a block, each of the six display types (three display sizes $\times$ target present/absent) were presented eight times, with order of presentation randomized. Each subject received 2 blocks of practice trials before performing the experiment proper. When the practice blocks were completed, the subject performed 10 blocks of experimental trials during which data was collected. Therefore, each subject performed 480 experimental trials yielding 80 responses in each of the six cells (display size $\times$ response type) within a condition. The entire procedure, including practice, was completed in about $25 \mathrm{~min}$.

\section{Results}

In general, errors were very infrequent (averaging 2\% per subject) and were not analyzed further. A preliminary four-way (two between-subject and two withinsubject factors) analysis of variance (ANOVA) was performed on the reaction time (RT) data. The main effect of target type (vertical target in horizontal distractors vs. horizontal target in vertical distractors) was not significant and did not interact with any other factor or combination of factors. All other ${ }^{2}$ main effects and interactions were significant at the $p<.0001$ level. Figure 4 shows the significant three-way interaction relating $\mathrm{RT}$ to number of display items for both response types for the three groups. The qualitative results are clear. For both Conditions 1 and 3, the subjects were able to detect the disparate element in a time that was relatively unaffected by the number of background distractors, whereas in Condition 2, search time was much more dependent on the number of background distractors.

Because we were concerned with specific comparisons within the design, the experiment was analyzed using a set of orthogonal weights first with RT and second with slope-defined by a least squares fit-as the dependent measure. In the RT analysis, the target-type and displaysize factors were collapsed, and in the slope analysis, the target type factor was collapsed. The RT analysis served to compare the overall speed with which targets can be detected, and the slope analysis assessed the influence of display size independently of overall RT. In both analyses, the main effect of condition was encoded using linear and quadratic terms. Then, within each condition, response types (target present/target absent) were compared.

An ANOVA of the RT data revealed a significant groups effect $[F(2,33)=57.57, p<.0001]$, which was made up of a linear component, indicating that the average RT in Condition $1(550 \mathrm{msec})$ was significantly faster $[F(1,33)=4.42, p<.05]$ than that in Condition 3 $(633 \mathrm{msec})$ and a quadratic term, indicating that the average RT in Condition 2 was significantly longer (954 msec) than those in Conditions 1 and 3 combined. Within each condition, RTs for target-present and target-absent trials were compared and found to be significantly different in all three cases $[F(1,11)=8.96,95.34$, and 34.46 , all $p$ s $<.05$, for Conditions 1,2 , and 3 , respectively], reflecting faster target-present than target-absent decisions.

A second ANOVA was performed on the slopes relating search times to number of display items for targetpresent and target-absent trials in all three conditions. The 
Condition 1

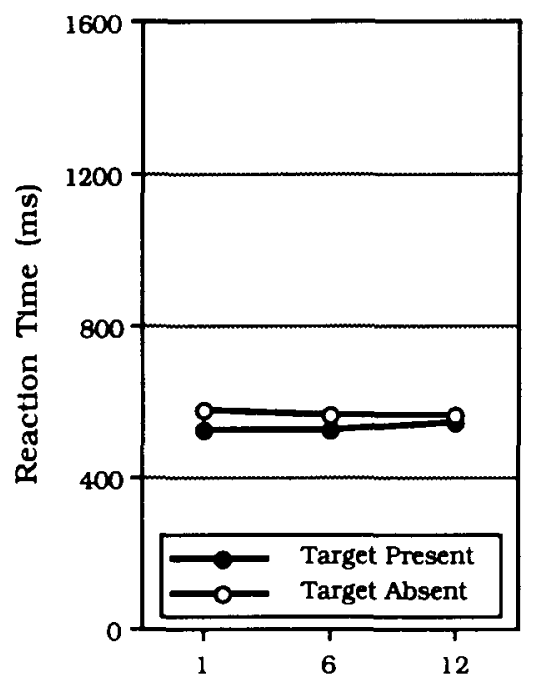

Number of Items
Condition 2

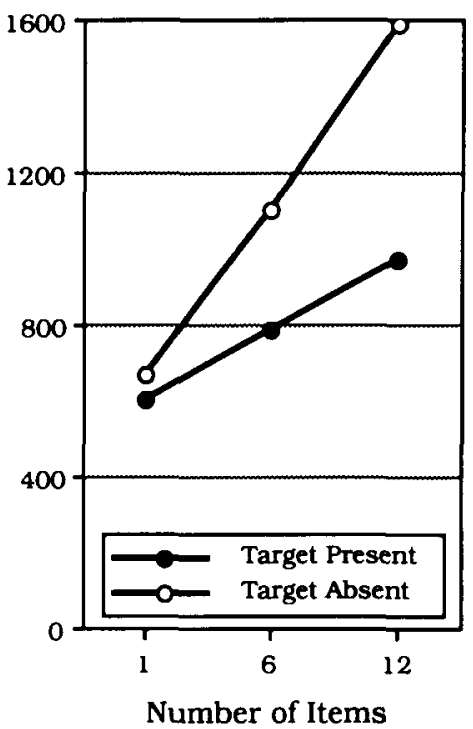

Condition 3

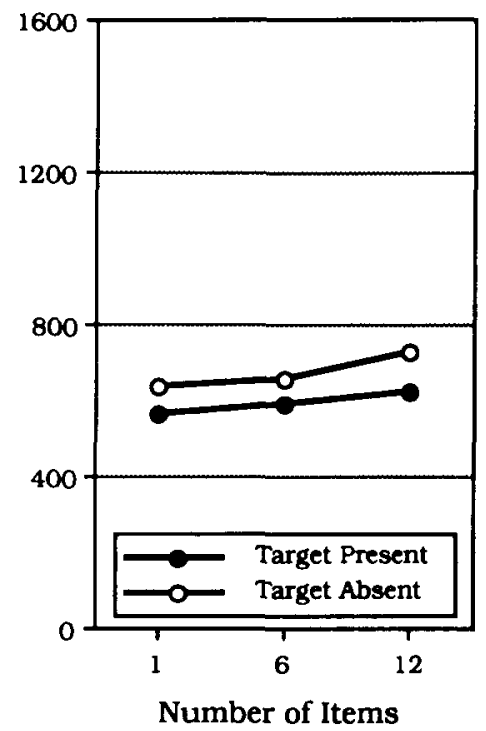

Figure 4. Summary of the three conditions in Experiment 1A. Each panel shows mean reaction times for each of the three conditions as a function of the number of display items and whether the target was present or absent. The principal result is that Conditions 1 and 3 yield flat functions for both target-present and target-absent trials, whereas Condition 2 yields a strong dependency on the number of items in the display.

RT slopes (averaged over target-present and target-absent trials) for Condition $1(0.11 \mathrm{msec} / \mathrm{item})$ were not significantly different $[F(1,33)=1.94, p>.15]$ from the $\mathrm{RT}$ slopes for Condition 3 (6.88 $\mathrm{msec} /$ item), whereas Condition 2 ( $58.39 \mathrm{msec} /$ item) was significantly different from the average of Conditions 1 and $3[F(1,33)=$ $169.55, p<.0001]$. The very shallow RT slopes for Conditions 1 and 3 are, by the standards of the visualsearch literature, indicative of parallel processing. That is, the addition of distractors had little effect on the speed with which the target is detected. Indeed, it should be noted that all subjects were naive to this sort of task and had no history of participation in psychophysical experiments. Other shallow search slopes reported in the literature and interpreted as reflecting parallel processing have been obtained from relatively practiced subjects using smaller numbers of display items (e.g., Duncan \& Humphreys, 1989).

The slope differences between target-present and targetabsent trials for Conditions 1 and 2 were significant $[F(1,11)=6.13$ and $75.35, p<.04$ and .0001 , respectively], although not for Condition $3[F(1,11)=3.87$, $p>$.05]. Such a difference is consistent with a serial search in Condition 2, but we attempt no interpretation of this effect for Condition 1.

In Condition 1 (luminance contours), the subjects were able to detect the disparate element in a time that was independent of the number of background distractors, a classic indicator of parallel search. In Condition 2, RT increased as a linear function of the number of background distractors and there was a much steeper search-time slope in the target-absent case, a classic indicator of serial search. In Condition 3, where the luminance/similarity competition provided by the circular frame (Condition 2 ) was eliminated, the search-time slope again became very flat, suggesting a parallel search. We may conclude from this experiment that orientation differences defined by subjective contours (as in Condition 3) can be detected in parallel when the subjective contours do not have to compete with actual luminance gradients (as in Condition 2). That is, attention does not need to be allocated serially to individual items to determine their orientation.

\section{EXPERIMENT 1B}

One could argue that in Condition 3 in Experiment 1A, the subjects were not detecting orientation differences on the basis of "spatial" subjective contours, but rather through short-range apparent motion (Braddick, 1975). In Condition 3, the sequence of a trial involved a pretrial display comprising the diagonal grating, and the presentation of the display items involved the vertical displacement of vertical or horizontal rectangular patches. This displacement might induce apparent motion. On the one hand, this interpretation would support the general notion that orientation differences are detected in parallel independent of the transmission medium (Cavanagh, Arguin, \& Treisman, 1990). However, such an interpretation confounds our attempt to study subjective contours. A task that directly assesses the role of temporal features involves presentation of a blank prefield prior to the presentation of the display materials. This was done in Ex- 
Blank Prefield

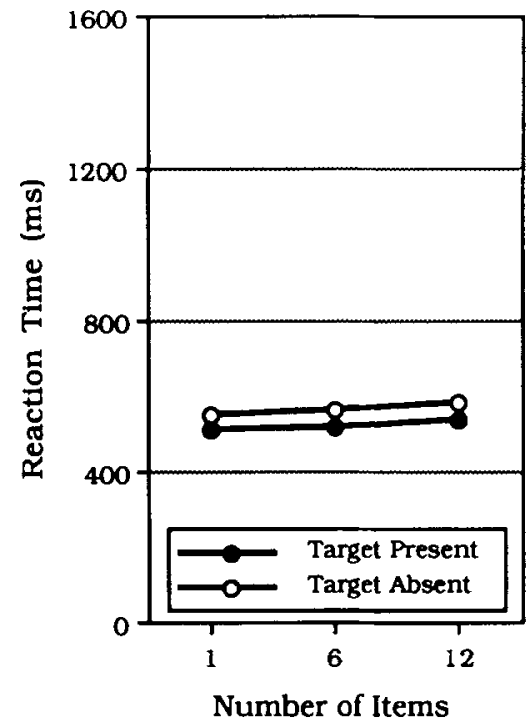

Grating Prefield

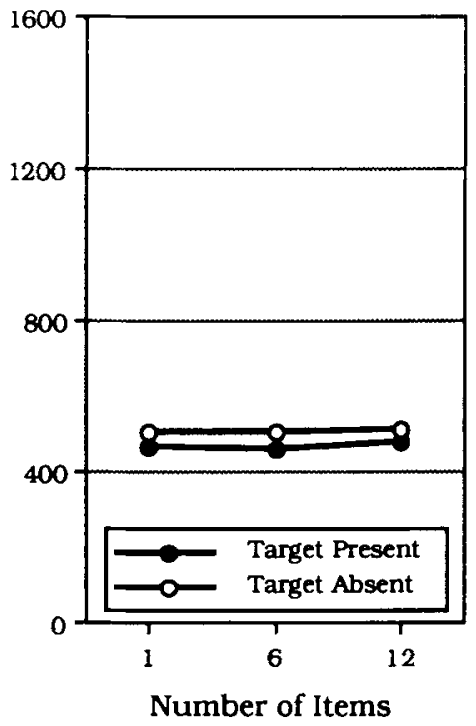

Figure 5. Summary of the two conditions in Experiment 1B. The right panel shows a replication of Condition 3 in Experiment $1 \mathrm{~A}$, in which the prefield was an entire field of diagonal lines (gratingprefield condition). The left panel shows the results of the blank-prefield condition, in which the prefield was a blank (white) screen. Overall reaction times are longer in the blank-prefield condition, but the search slopes remain flat.

periment $1 \mathrm{~B}$ using 6 subjects, 2 of whom were authors and 4 of whom were graduate students who had not previously participated in this experiment. Each subject performed 480 trials in the grating-prefield condition, which was the same as Condition 3 in Experiment $1 \mathrm{~A}$, and an additional 480 trials in the blank-prefield condition, which was identical in all respects except that a blank (white) prefield was presented prior to the test materials. That is, the background and items appear simultaneously. Order of presentation was counterbalanced so that 3 subjects performed the new task (blank-prefield condition) first and 3 performed the old task (grating-prefield condition) first.

\section{Results}

The results are summarized in Figure 5. Overall, RT was slower in the blank-prefield condition than in the grating-prefield condition $[F(1,5)=70.69, p<.005]$ and target-present trials were faster than target-absent trials $[F(1,5)=27.92, p<.005]$. However, the RT slopes in the grating-prefield condition $(.83 \mathrm{msec} /$ item) were not significantly different from the RT slopes in the blank-prefield condition $(2.61 \mathrm{msec} / \mathrm{item})[F(1,5)=$ $4.681, p>.09$ ]. Thus, search was parallel whether or not the displays contained transient information. We interpret the mean RT elevation in the blank-prefield condition to reflect the masking effects of the blank prefield. The transition from the blank prefield to the display materials represents spatiotemporal noise that slows encoding of the subjective contours.

\section{EXPERIMENT 2}

The stimuli of Experiment 1A may be considered somewhat nonstandard in that we have used subjective contours induced by gratings whose components are oblique to the orientations of the contours, whereas in classical demonstrations of the effect the inducing gratings have been orthogonal (or nearly so) to the orientations of the subjective contours. Experiment 2 was designed along similar lines to Condition 3 in Experiment $1 \mathrm{~A}$ with the exception that the targets and distractors were vertical bars and vertically oriented crescents defined by horizontal black lines (grid) displaced with respect to a background grid that also comprised horizontal black lines. Figure 6 " shows examples of these stimuli. It would be expected that search should be relatively independent of the number of background distractors.

\section{Method}

Subjects. Fifteen naive subjects were drawn from the undergraduate subject pool of the Psychology Department at the University of Western Ontario. All subjects reported normal or corrected-to-normal vision. The subjects received partial course credit for their participation.

Displays. Examples of the stimuli are shown in Figure 6. The horizontal grating was composed of 1-pixel-wide lines with a cycle of 4 pixels. The vertical bars and crescents were constructed by displacing the form by half a cycle in the vertical direction. The vertical bars were 52 pixels $(20 \mathrm{~mm})$ in the vertical direction and 15 pixels $(5.5 \mathrm{~mm})$ in the horizontal direction, and the crescents were 52 pixels $(20 \mathrm{~mm})$ in the vertical direction and 21 pixels $(8 \mathrm{~mm})$ in the horizontal direction (i.e., maximum horizontal extent). 


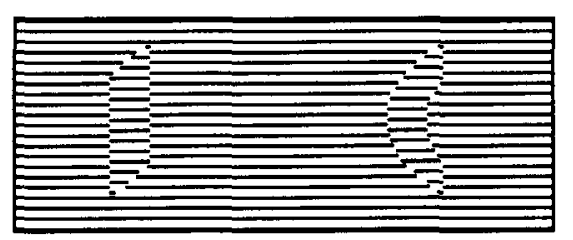

Figure 6. Examples of the stimuli used in Experiment 2. The subjective contours shown here are more like the classical examples than those in Experiment $1 \mathrm{~A}$.

Procedure and Apparatus. The procedure and apparatus were exactly as in Condition 3 in Experiment $1 A$ (there was no blank pretest field) except for the figures involved. Seven subjects searched for a crescent among vertical bars, and 8 subjects searched for a vertical bar among crescents.

\section{Results}

One subject in the group searching for a vertical bar among crescents showed an unacceptably high error rate $(10 \%)$ and was therefore dropped from the analysis, leaving 7 subjects in each group. Thirteen of the remaining subjects had error rates of less than $3 \%$, and 1 had an error rate of $4.5 \%$. The results of Experiment 2 are summarized in Figure 7. A simple, two-factor ANOVA was performed on both the RT and slope data with target type (vertical bar vs. crescent) as a between-subject factor and response type (target present vs. target absent) as a withinsubject factor.

The RT analysis revealed a significant groups (target type) effect $[F(1,12)=9.802, p<.01]$. Search was faster when a crescent was the target than when the vertical bar was the target, that is, there was a search asymmetry. Response type was also significant $[F(1,12)=74.814, p<.001]$, indicating that responses

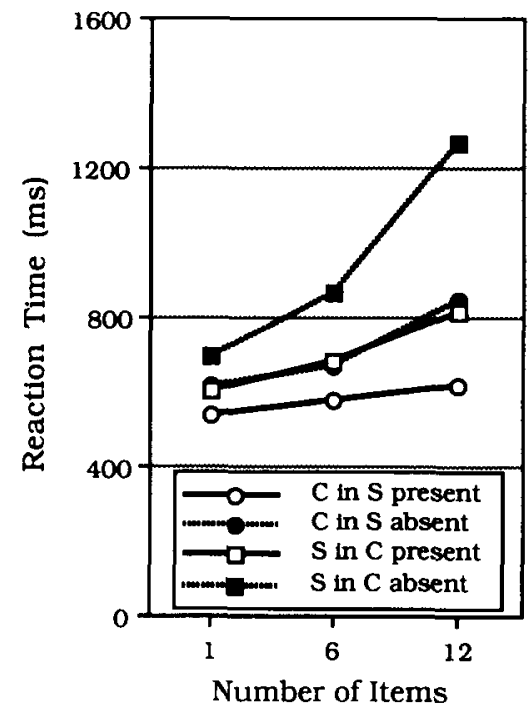

Figure 7. Summary of the two conditions in Experiment 2. Search rates are faster when subjects are searching for a crescent $(C)$ in vertical bars (S), both for the target-present and target-absent conditions, that is, there is a search asymmetry. on target-present trials were faster than on target-absent trials. There was also a significant interaction $[F(1,12)=$ $6.329, p<.03$ ], indicating that the difference between target-present and target-absent responses was greater for vertical targets than for crescent targets.

A second ANOVA was performed on the search slopes using the same factors as in the previous RT analysis. There was a main effect of groups $[F(1,12)=12.88$, $p<.005]$, indicating that search rate, averaged over response type, was faster when crescents $(14.08 \mathrm{msec} / \mathrm{item})$ were targets than when vertical bars $(35.76 \mathrm{msec} / \mathrm{item})$ were targets. There was also a main effect of response type $[F(1,12)=34.72, p<.0005]$, indicating that the presence of a target was detected more quickly than its absence. The interaction of groups $\times$ response type was also significant $[F(1,12)=5.26, p<.04]$, indicating that the slope difference between target-present and targetabsent trials was greater when vertical bars were the targets.

Experiment 2 partially corroborates the results of Experiment 1A. It was expected that search for the disparate elements should be relatively unimpaired by the addition of distractors in the present experiment, but it was found that this was only true when the subjects were searching for a crescent in vertical bars $(6.9 \mathrm{msec} / \mathrm{item}$ in the target-present case). The change in the form discrimination to be made (from horizontal and verticals to vertical bars and crescents) seems to have had an interesting effect on the results. Although the search rate for crescents is generally within the preattentive range, the search rate for vertical bars in crescents $(19.4 \mathrm{msec} / \mathrm{item})$ is not. The appropriate conclusion seems to be that subjective contours defined by offset gratings whose constituent lines are orthogonal (or nearly so) to the contours they define can be discriminated in parallel (i.e., in a time that is essentially independent of the number of background distractors). However, this discrimination is dependent on the target-distractor relationship. Such detection and discrimination asymmetries are common in the visual-search literature (Frith, 1974; Treisman \& Gormican, 1988; Treisman \& Souther, 1985) and in the texture-discrimination literature (Gurnsey \& Browse, 1987, 1989; Rubenstein \& Sagi, 1990). They may be interpreted as reflecting a Weber-like relationship between stimuli that vary along a common dimension (Gurnsey \& Browse, 1989, Experiment 1; Treisman \& Gormican, 1988) or in terms of response uncertainty due to noise (Gurnsey \& Browse, 1989, Experiment 2; Rubenstein \& Sagi, 1990). (We will return at length to this point in the General Discussion.) Therefore, the fast search rate for crescents in vertical bars and the search asymmetry in the present experiment are both consistent with the idea that subjective contours are registered at a very early stage in the visual system.

\section{EXPERIMENT 3}

It has been adequately demonstrated in the preceding experiments that the targets in our displays are detected in the preattentive range. A question arises, however, 


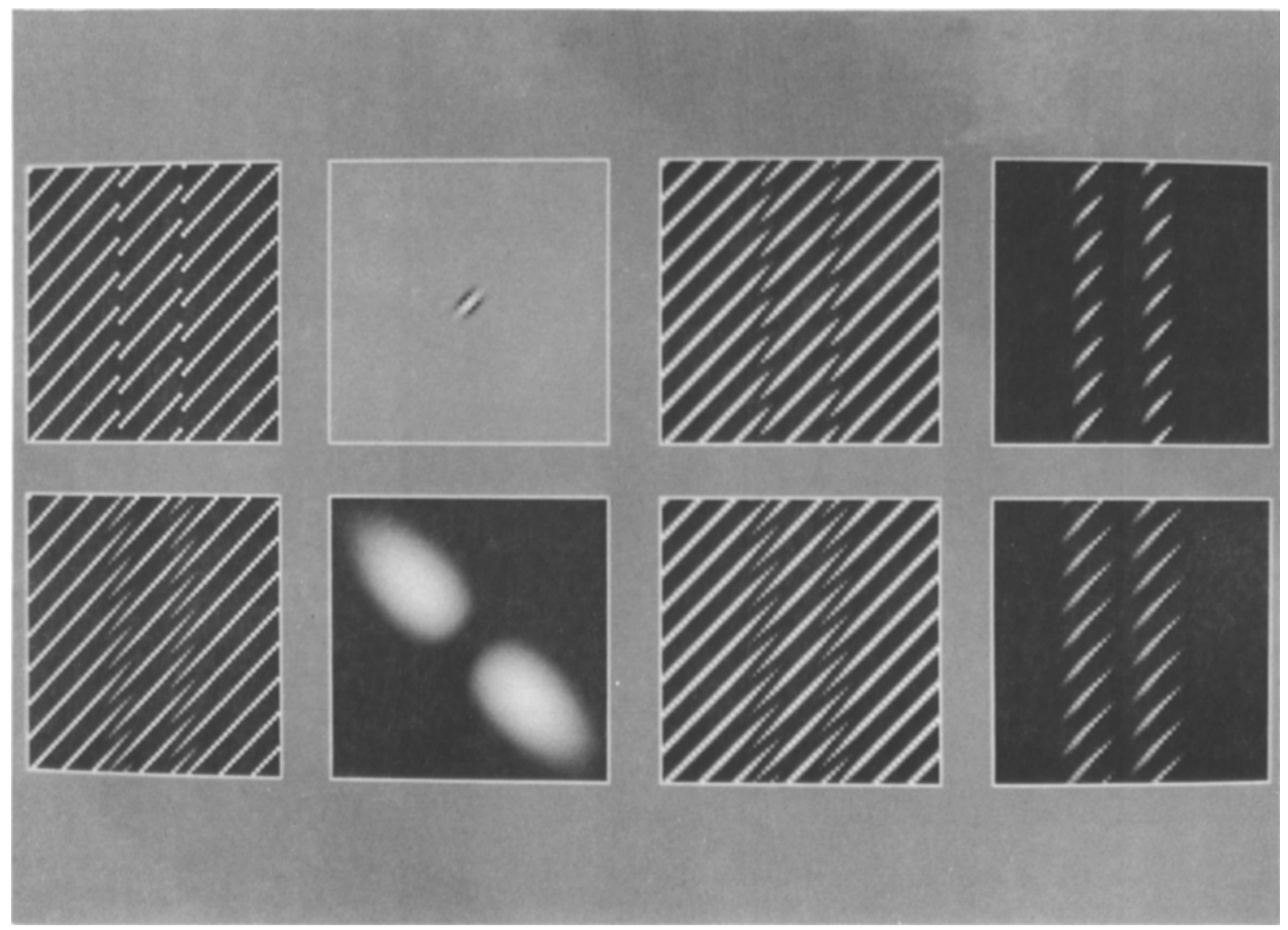

Figure 8. The top and bottom panels of the left column show examples of contours similar to those used in Experiment 3; the actual stimuli involved black lines on a white background. The top panel of the next column shows an orientation-selective unit (with peak sensitivity at $\mathbf{4 5}^{\circ}$ ). The unit is defined in terms of three offset Gaussian distributions, in each of which the major axis has a standard deviation $(\sigma)$ three times that of the minor axis. The three Gaussians are offset along the minor axis by an amount equal to one $\sigma$ along the minor axis. The operator represents the weighted sum of the response of each Gaussian, where the flanking regions are given a weight of -1 and the central region is given a weight of 2 . The panel below shows its Fourier amplitude spectrum. The top and bottom panels of the next column (second from the right) show the half-rectified convolution of the orientation-selective unit with the top and bottom panels, respectively, of the left column. The top and bottom panels of the right column show the effects of end stopping. This was achieved by simply taking the difference of two points in the convolution output displaced by a small amount in the direction of the main axis (45 ${ }^{\circ}$ ) of the orientation-selective unit. The contrast of the response at the border is greater in the top panel (abrupt terminations) than in the bottom panel (tapered terminations). Although it may not be striking in the reproduction, the maximum response in the top panel is twice that in the bottom panel. If end-stopped operators are involved in the detection of subjective contours (as suggested by von der Heydt \& Peterhans, 1989), then we would expect that tapering the terminations would attenuate the responses of such operators at the boundary, as shown, and consequently impair detection of the contour.

about what aspect of the display our subjects are responding to. It is generally thought that subjective contours induced by displaced lines are critically dependent on aligned, abrupt terminations (Grossberg \& Mingolla, 1985; Kennedy, $1988^{3}$ ). If this is so, then we would expect that registration of subjective contours would be impaired if the line terminations were tapered rather than abrupt (see Figure 8). On the other hand, if the subjects were responding to some other aspect of the display (e.g., the phase difference between the lines inside and outside of the bars), then there would be no reason to expect line tapering to have any effect. Bravo and Blake (1990), for example, found parallel search for orientation differences defined by texture boundaries. A local phase shift in the diagonal grating (as in our experiments) might be thought of as a texture difference that carries form information that does not crucially depend on the abruptness of the line terminations. In our final experiment, we examined this question explicitly. For technical reasons, the visualsearch paradigm could not be used. Therefore, we addressed this issue in a texture-discrimination paradigm in which subjects were to localize a $3 \times 4$ region of horizontal bars (defined by subjective contours) within a larger background of horizontal bars (and vice versa). In one condition, the horizontal and vertical bars were exactly the same as those used in Experiments 1A and 1B. In three other conditions, the line terminations were tapered rather than abrupt (Figure 8). The approach in Experiment 3 is to ob- 
tain a psychometric function that relates discriminability to degree of tapering for a few relatively experienced subjects.

\section{Method}

Subjects. Three subjects participated in the experiment. One subject was one of the authors, and the other 2 were psychology graduate students.

Displays. A textural display comprised a 10 (horizontal) $\times 7$ (vertical) array of horizontal and vertical bars whose dimensions were identical to those used in Experiment 1A. Each bar was slightly jittered about the center of its array location to eliminate large-scale grouping effects. On a given trial, a $3 \times 4$ region of vertical (or horizontal) bars was embedded in one quadrant of the display in a larger background of horizontal (or vertical) bars. Each bar was defined within a $64 \times 64$ array of pixels $(24 \times 24 \mathrm{~mm})$. As before, the viewing distance was approximately $50 \mathrm{~cm}$. The entire display, then, had the appearance of horizontal and vertical bars offset within a homogeneous background of diagonal lines (essentially as in Experiment $1 \mathrm{~A}$ ).

In the no-blur condition, the horizontal and vertical bars were identical to those used in Experiment $1 \mathrm{~A}$, that is, horizontal and vertical bars defined by offsetting a rectangular region of a diagonal grid by half a cycle vertically. The three blur conditions were created from the nonblurred stimuli by setting each pixel in the array to be the unweighted average of itself and its diagonal neighbors placed symmetrically about it. (Of course, this only has an effect in the region of a termination.) Three degrees of blurring were created by varying the neighborhood size in which the averaging was done, that is, a region 3,5 , or 7 pixels wide. The color look-up table of the monitor was calibrated to be linear (using a Hagner photometer). This ensured that there would be no intensity gradient aligned with the direction of the contour when moving from the interior to the exterior of one of the bars.

Apparatus and Procedures. All aspects of display and data collection were under the control of an Apple Macintosh II computer. Before each trial, the subjects were presented with a blank screen having a small, centrally placed fixation dot. When ready, the subjects pressed a button to initiate a trial. The fixation screen was immediately replaced with the textural display, which remained on for a predetermined time. The offset of the texture display was followed by $500 \mathrm{msec}$ of blank screen, which in turn was followed by the presentation of four unfilled rectangles, one in each quadrant of the display. The subjects moved a mouse-controlled pointer into one of the rectangles and clicked to indicate where they judged the disparate region to have been. They were instructed to guess if uncertain. If the response was correct, then the rectangle was changed to solid black; otherwise it remained unfilled. Following this, the fixation screen reappeared and the sequence repeated.

Preliminary testing showed individual differences in general sensitivity in this task. Therefore, to keep performance in the same range, the subjects were run at different exposure durations. For Subject R.G., the exposure duration of the textural displays was set at $30 \mathrm{msec}$, for A.H. at $60 \mathrm{msec}$, and for B.P. at $120 \mathrm{msec}$.

Each subject was presented with eight different textural displays (four degrees of blurring for both horizontals in verticals and vice versa). For each of the eight display types, the disparate texture could appear in one of the four quadrants of the display. A block consisted of four replications of the factorial combination of display type and quadrant for a total of $8 \times 4 \times 4=128$ trials. Presentation was randomized within a block. Within a session, each subject was presented with four blocks of trials for a total of 512, representing 128 responses for each degree of blurring.

\section{Results}

Figure 9 summarizes the effect of blurring for each of the three subjects. The results are collapsed over target

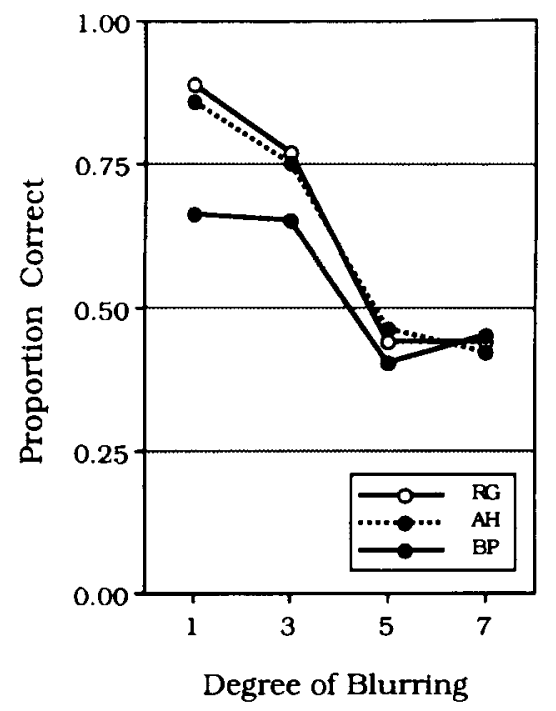

Figure 9. Summary of the discriminability of the horizontal and vertical bars as a function of degree of tapering for Subjects R.G., A.H., and B.P. averaged over horizontals in verticals and verticals in horizontals. The exposure duration was $30 \mathrm{msec}$ for R.G., $60 \mathrm{msec}$ for A.H., and 120 msec for B.P. Performance is best when lines terminate abruptly and falls off monotonically as the terminations are increasingly blurred (i.e., tapered). This suggests that abrupt terminations are important to the detection of subjective contours and may implicate the sort of end-stopped mechanism described in Figure 8.

type, that is, verticals in horizontals and horizontals in verticals. A clear effect of blurring is evident for all three subjects such that performance dropped as the terminations became increasingly blurred, this is, performance was best overall when line terminations were abrupt. To assess the statistical reliability of these data, they were subjected to a one-factor ANOVA, and the main effect of blurring was significant $[F(3,2)=32.41, p<.0005]$. This main effect was made up of a significant linear trend $[F(1,2)=29.53, p<.05]$, indicating a general decrease in performance with increased blurring; a significant quadratic trend $[F(1,2)=20.16, p<.05]$, indicating that performance decrement was not monotonic with degree of blurring but evened out at levels 5 and 7 ; and a significant cubic trend $[F(1,2)=188.44, p<.01]$, which seems to reflect the sharp step between the intermediate levels of blurring.

The question addressed by Experiment 3 was whether the abruptness of the terminations aligned along subjective contours was important to their detection and discrimination. Ideally, this would have been done in the search paradigm utilized in Experiments $1 \mathrm{~A}$ and 2. Technical limitations precluded this, so we utilized a four-alternative, forced-choice procedure in a texture-discrimination paradigm. The results clearly indicate that the abruptness of line terminations is related to the ease with which textures can be discriminated. We conclude that a stronger orientation signal is induced by the abrupt terminations than by the tapered terminations. This result corroborates the demonstrations of Kennedy (1988). Given the brief 
exposures in our displays and the uncertainty about where, and what, materials would be presented on a particular trial, it is reasonable to conclude that the stimuli were engaging low-level visual mechanisms and that the subjects were basing their discriminations rather directly on the outputs of these mechanisms.

\section{GENERAL DISCUSSION}

The results of the three experiments described here demonstrate that (1) orientation and form differences between figures defined by subjective contours can be detected in a time that is independent of the number of background distractors (Experiment 1A, Condition 3; Experiment 2, crescents in vertical bars); (2) detection of these differences can be impaired by other aspects of the display that provide competing organizations (Experiment $1 \mathrm{~A}$, Condition 2); (3) in certain cases, form differences defined by subjective contours can give rise to discrimination asymmetries; (4) textures comprising items defined by subjective contours can be discriminated very quickly; and (5) if the aligned terminations that define the subjective contours are tapered, discrimination is impaired relative to cases in which the line terminations are abrupt.

The principal motivation for the present experiments was to determine if forms defined by subjective contours are discriminated independently of the number of background distractors. The results show clearly that this is so, but that this discrimination can be impaired by competing forms elsewhere in the display. Therefore, these results are consistent with our proposal that the failure to find "preattentive" discrimination in previous studies (Grabowecky \& Treisman, 1989; Meyer \& Fish, 1987) was due to the presence of competing form information elsewhere in the display.

There remain, however, the results of Pritchard and Warm (1983). They showed that same-different judgments based on subjective contours were more seriously impaired by added attentional load than were judgments based on real contours. Their study involved three stimulus conditions: (1) subjective contours defined by Kaniza-type pacmen, (2) the same pacmen with the contours filled in with thin black lines, and (3) the entire figure defined by brightening the pacmen. Thus, there were two versions of the real contour figures and one case of subjective contours. Pritchard and Warm found a significant main effect of attentional load over the three stimulus conditions used, that is, adding an attentional load slowed RTs. Pritchard and Warm's Table 1 shows mean RTs for conditions of attentional load and no attentional load, as a function of same and different judgments, in three test blocks, for all three stimulus conditions. In all 18 cases ( 3 stimulus types $\times 3$ test blocks $\times 2$ response types), RTs were faster when there was no attentional load. Although a post hoc test revealed that only same judgments for subjective contours differed statistically as a function of attentional load, there are sufficient grounds for believing that there is a general attention effect that would be revealed by more sensitive procedures. If this is so, then the difference between real and subjective contours is one of degree and not kind and the Pritchard and Warm data do not distinguish between processing of real and subjective contours on the basis of attention.

It would be expected that same-different judgments for shapes defined purely by luminance contrast would become harder as the contrast is reduced. Subjects would have to devote more attentional resources to make the discrimination even though the initial registration of the border contrast could be explained in terms of the responses of cells in the striate cortex, that is, attention would be applied to the outputs of those cells, perhaps to improve the signal-to-noise ratio. So, it is decision stages that involve attention, not the construction of the representation. Given that subjective contours may be considered to be like low-contrast real contours, then considerable impact would be removed from the Pritchard and Warm (1983) results if it were found that samedifferent judgments for real contours interacted with attentional load as the boundary contrast is reduced. Such an outcome would be consistent with the present results and remains a point for future study.

An unpredicted result of Experiment 2 was the finding that discrimination of forms defined by subjective contours can produce asymmetrical search rates. Thus, finding a crescent in a background of vertical bars produces shallower search slopes than does the obverse case. Treisman and Gormican (1988) reported similar results with curved and straight line segments. There are two ways (at least) that discrimination asymmetries have been explained in the past. We refer to these accounts as the Weber and noise explanations and deal with each in turn.

The Weber explanation has been proposed to account for discrimination asymmetries in both visual search (Treisman \& Gormican, 1988) and texture discrimination (Gurnsey \& Browse, 1989), although in somewhat different ways. Fundamental to the explanation is the assumption that in either visual search or texture discrimination, the items involved can be considered to represent points on what has been classically called a prothetic dimension. Luminance is a prothetic dimension because it is some, thing that an element in a display can have more or less of. Thus, in visual search or texture discrimination, we can consider the two elements involved to have magnitudes $a$ and $b$ on some prothetic dimension such that $a>b$. In the case of texture discrimination, where one is to localize a "foreground" region of $n_{\mathrm{fg}_{\mathrm{g}}}$ textural elements in a larger background of $n_{\mathrm{bg}}$ different textural elements (i.e., $n_{\mathrm{bg}}>n_{\mathrm{fg}}$ ), it is clear that the foreground/background textural contrast will be greater when the elements having magnitude $a$ form the foreground. That is,

$$
n_{\mathrm{fg}} a / n_{\mathrm{bg}} b>n_{\mathrm{fg}} b / n_{\mathrm{bg}} a .
$$

This observation obviously generalizes to the case of visual search, ${ }^{4}$ where $n_{\mathrm{fg}}=1$.

According to such an analysis, then, one needs to identify the dimension along which stimuli are varying. Treisman and Gormican (1988) suggest that curvature is a 
dimension that conforms to this analysis. Each element in the display can be characterized in terms of its degree of curvature. In Experiment 2, the vertical bars have a low degree of curvature and the crescents have a higher degree of curvature. Therefore, from the above expression, it is clear that a single crescent should contrast more strongly with a background of vertical bars than vice versa.

The noise explanation has been proposed as an alternative account of discrimination asymmetries and can be related to the work of Gurnsey and Browse (1989), Rubenstein and Sagi (1990), Duncan and Humphreys (1989), and Pavel (1990). The most intuitive account can be framed roughly in terms of the work of Rubenstein and Sagi. Rubenstein and Sagi would argue that the ease with which a target can be detected in a visual-search task is dependent both on the similarity between the target and background distractors and the similarity between distractors. Following from this analysis, an average difference, $d$, between targets and distractors can be made easy or hard to detect by manipulating the interitem similarity among the distractors. It may be easy to detect $d$ if the distractors are homogeneous but difficult if they are heterogeneous. In the case of asymmetric visual search, the difference between targets and distractors is always fixed. However, if one set of elements is more variable than the other, this fixed difference, $d$, will be more difficult to detect when the background distractors are heterogeneous than when they are homogeneous.

According to this analysis, one has to identify the difference involved and a measure of variability. In the case of curvature - and to simplify we will first consider curvature in the luminance domain-assume that detection in a visual-search task comes about from differences in the outputs of orientation and scale-selective filters. An important point, according to this analysis, is that an image curve can be thought to comprise a number of piecewise linear segments. Therefore, a curve (crescent) would elicit responses from a large number of orientationselective units, whereas a vertical bar would elicit responses principally from units selective for vertical contrast. It then follows that there will be differences in orientation responses between adjacent crescents, and even within crescents. The important point is that there will be orientation contrast between target and distractors whether a crescent is a target and vertical bars are distractors or vice versa; however, there will only be orientation contrast between distractors when the crescents form the distractors and a vertical bar is the target. The orientation contrast between distractors would then be noise that competes with the contrast between target and distractors (Rubenstein \& Sagi, 1990). Of course, this explanation can be generalized from the luminance domain to subjective contours both in principle and in fact because of the observations of von der Heydt and Peterhans (1989).

Faced with these two models of discrimination asymmetry, we tend to favor the noise model for several reasons. First, cells in V2 have been found to respond selectively to the orientation of subjective contours, but it is unclear whether they respond to curvature in a similar manner. Second, such an analysis fits well with the computational model of Rubenstein and Sagi (1990), which provides an excellent fit to existing data on discrimination asymmetries. Thus, the current data may reflect an instance of the same general phenomena considered by Rubenstein and Sagi. Finally, we are reluctant to posit maps that make explicit the degree to which curvature is present at each point in the image. We are currently exploring a computational model of a mechanism selective for the orientation of subjective contours and hope to relate its behavior to the asymmetries seen in Experiment 2 in a manner that follows the analysis of Rubenstein and Sagi and Pavel (1990).

Experiment 3 utilized subjective contours in the context of a texture-discrimination paradigm. Since these are novel stimuli for this sort of task, we will conclude our discussion with a brief comment about the relationship of Experiment 3 to existing models of texture discrimination.

The complexity of a visual computation is often indexed by the speed with which it can be carried out. Therefore, brief presentations have been used to determine if the features which comprise two textures are registered at an early stage of the visual system (Gurnsey \& Browse, 1987, 1989; Julesz, 1981, 1984). We have shown in Experiment 3 that very brief exposures are sufficient to establish the conditions necessary to discriminate two textures whose elements are defined by subjective contours. Therefore, this discrimination would seem to involve very simple mechanisms. Several qualitative and quantitative models of visual texture discrimination have been proposed in recent years (Bergen \& Landy, 1990; Fogel \& Sagi, 1989; Gurnsey \& Browse, 1989; Malik \& Perona, 1990; Rubenstein \& Sagi, 1990), all of which share the assumption that two stages of filtering are applied to find textural discontinuities. The first stage involves the application of linear, orientation- and scale-selective filters at each point in the array. This stage is motivated by the many studies showing that $\mathrm{V} 1$ acts in this general way to produce a large number of "neural images" (Robson, 1980 ), each with particular orientation and scale tuning. A second stage of filtering is applied to each neural image to localize "texture energy differences."

As noted several times already, orientation selectivity for subjective contours exists in many V2 cells. Those cells do double duty because they are also selective for luminance contours of roughly the same orientation. If the mechanisms responsible for the discrimination of textures involving subjective contours are the same as those involved in the discrimination of elements defined in the luminance domain, then a plausible conclusion is that texture gradients are computed in terms of a somewhat more processed representation such as exists at $\mathrm{V} 2$ rather than at V1, as is tacitly assumed in existing models. Furthermore, if V2 cells respond to subjective contours by in- 
tegrating the outputs of end-stopped simple cells, this would explain why blurring the line terminations in Experiment 3 disrupted the discrimination of the two textures.

The research described in this paper was motivated by a desire to resolve a perceived contradiction in the literature: On the one hand there is compelling evidence that subjective contours are the products of reflexive, low-level visual processes, but there are also equally compelling reasons to suspect the involvement of more deliberative processes. Our results are consistent with the proposal that subjective contours are registered reflexively, but because of competing luminance contours, for example, attention may be required to gain access to them. This is but a piece in the puzzle of subjective contours. A number of questions remain. For example, not all image structures that are referred to as subjective contours elicit the same perceptual judgments or respond in similar ways to experimental manipulations (Parks \& Marks, 1983; Richardson, 1979), suggesting different neural origins. Also, if, as we have tacitly accepted, subjective contours are encoded by the same mechanisms that encode luminance contours, then some explanation has to be given for why real and certain subjective contours fragment differently (Halpern \& Warm, 1980). Therefore, the tasks and controls used in the present research may be applied to other sorts of subjective contours to determine if they elicit similar behaviors.

In conclusion, our results show that certain subjective contours are registered in a bottom-up, or reflexive, manner. Perception of-or access to-this low-level representation may require further processes that make use of other sources of information that are present in the scene (e.g., Rock \& Anson, 1979) or in memory (Wallach \& Slaughter, 1988). Thus, we see no necessary contradiction between our findings and interpretation and those of others that indicate a role for interpretive and/or memorial factors in the perception of subjective forms.

\section{REFERENCES}

BECK, J. (1982). Textural segmentation. In J. Beck (Ed.), Organization and representation in perception (pp. 256-317). Hillsdale, NJ: Erlbaum.

BERGEN, J. R., \& LANDY, M. S. (1990). Computation modeling of visual texture segregation. (Unpublished manuscript.)

BRADDICK, O. J. (1975). A short range process in apparent motion. Vision Research, 14, 519-527.

Bravo, M., Blake, R. (1990). Preattentive vision and perceptual groups. Perception, 19, 515-522.

CAELLI, T. M. (1980). Facilitative and inhibitory interactions in visual texture discrimination. Biological Cybernetics, 39, 21-26.

Cavanagh, P., Arguin, M., Treisman, A. (1990). Effect of surface medium on visual search for orientation and feature size. Journal of Experimental Psychology: Human Perception \& Performance, 16, 479-491.

Duncan, J., Humphreys, G. W. (1989). Visual search and stimulus similarity. Psychological Review, 96, 433-458.

EnNs, J. T., OChs, E. P., \& Rensink, R. A. (1990). VSearch: Macintosh software for experiments in visual search. Behavior Research Methods, Instruments, \& Computers, 22, 118-122.
FoGEL, I., \& SAGI, D. (1989). Gabor filters as texture discriminator. Biological Cybernetics, 61, 103-113.

Fox, J. (1978). Continuity, concealment and visual attention. In G. Underwood (Ed.), Strategies in information processing (pp. 23-66). New York: Academic Press.

Fox, J., MAYHEW, J. E. W. (1979). Texture discrimination and the analysis of proximity. Perception, 8, 75-91.

FrITH, U. (1974). A curious effect with reversed letters explained by a theory of schema. Perception \& Psychophysics, 16, 113-116.

Grabowecky, M., \& Treisman, A. (1989). Attention and fixation is subjective contour perception. Investigative Ophthalmology \& Visual Science, 30(4), 457. (Abstract)

GrossberG, S., \& Mingolla, E. (1985). Neural dynamics of perceptual grouping: Textures, boundaries, and emergent segmentations. Perception \& Psychophysics, 38, 141-171.

Gurnsey, R., \& Browse, R. A. (1987). Micropattern properties and presentation conditions influencing visual texture discrimination. Perception \& Psychophysics, 41, 239-252.

Gurnsey, R., \& Browse, R. A. (1989). Asymmetries in visual texture discrimination. Spatial Vision, 4, 31-44.

HALPERN, D. F., \& WARM, J. S. (1980). The disappearance of real and subjective contours. Perception \& Psychophysics, 28, 229-235.

JULESZ, B. (1981). Textons, the elements of texture discrimination, and their interactions. Nature, 290, 91-97.

Julesz, B. (1984). Toward an axiomatic theory of preattentive vision. In G. Edelman, W. Einer, \& W. Cowan (Eds.), Dynamic aspects of neocortical function (pp. 585-612). New York: Wiley.

Kato, H., Bishop, P. O., \& ORBaN, G. A. (1978). Hypercomplex and simple/complex cell classifications in cat striate cortex. Journal of Neurophysiology, 41, 1071-1095.

Kennedy, J. M. (1988). Line endings and subjective contours! Spatial Vision, 3, 151-158.

Malik, J., Perona, P. (1990). Preattentive texture discrimination with early vision mechanisms. Journal of the Optical Society of America A, 7, 923-932.

MARR, D. (1982). Vision. San Francisco: W. F. Freeman.

MEYeR, G. E., \& FiSH, D. (1987). Illusory contours, texture segregation, and a configural inferiority effect. In S. Petry \& G. E. Meyer (Eds.), The perception of illusory contours (pp. 224-234). New York: Springer-Verlag.

NeIsSER, U. (1967). Cognitive psychology. Englewood Cliffs, NJ: Prentice-Hall.

Paradiso, M. A., Shimojo, S., \& Nakayama, K. (1989). Subjective contours, tilt aftereffects, and visual cortical organization. Vision $R e-$ search, 29, 1205-1213.

Parks, T. E., \& Marks, W. (1983). Sharp-edged vs. diffuse illusory circles: The effects of varying luminance. Perception \& Psychophysics, 33, $172-176$.

PAvel, M. (1990, November). A statistical model of preattentive visual search. Paper presented at the meeting of the Psychonomic Society," New Orleans.

Peterhans, E., \& von der Heydt, R. (1989). Mechanisms of contour perception in monkey visual cortex: II. Contours bridging gaps. Journal of Neuroscience, 9, 1749-1763.

Petry, S., \& Meyer, G. E. (1987). The perception of illusory contours. New York: Springer-Verlag.

Pritchard, W. S., \& WARM, J. S. (1983). Attentional processing and the subjective contour illusion. Joumal of Experimental Psychology: General, 112, 145-175.

ReDIEs, C. (1989). Discontinuities along lines: Psychophysics and neurophysiology. Neuroscience \& Biobehavioral Reviews, 13, 17-22.

RichardSON, B. L. (1979). The nonequivalence of abrupt and diffuse illusory contours. Perception, 8, 589-593.

RoBson, J. G. (1980). Neural images: The physiological basis of spatial vision. In C. S. Hams (Ed.), Visual coding and adaptability (pp. 177-214). Hillsdale, NJ: Erlbaum.

Rock, I. (1987). A problem solving approach to illusory contours. In S. Petry \& G. E. Meyer (Eds.), The perception of illusory contours (pp. 62-70). New York: Springer-Verlag.

Rock, I., ANSON, R. (1979). Illusory contours as the solution to a problem. Perception, 8, 665-681. 
Rubenstein, B. S., \& SAGI, D. (1990). Spatial variability as a limiting factor in texture-discrimination tasks: Implications for performance asymmetries. Journal of the Optical Society of America A, 7, 1632-1643.

SChNEIDER, W., \& SiFfrin, R. M. (1977). Controlled and automatic human information processing: 1. Detection, search and attention. Psychological Review, 84, 1-66.

SChumann, F. (1987). Contributions to the analysis of visual perception: First paper: Some observations on the combination of visual impressions into units. In S. Petry \& G. E. Meyer (Eds.), The perception of illusory contours. New York: Springer-Verlag. (Original work published 1900)

SMith, A., \& Over, R. (1975). Tilt aftereffects with subjective contours. Nature, 257, 581-582.

Smith, A. T., \& Over, R. (1979). Motion aftereffect with subjective contours. Perception \& Psychophysics, 25, 95-98.

Treisman, A. M., \& GeLADE, G. (1980). A feature-integration theory of attention. Cognitive Psychology, 12, 97-136.

Treisman, A., Gormican, S. (1988). Feature analysis in early vision: Evidence from search asymmetries. Psychological Review, 95 , $15-48$.

Treisman, A., Souther, J. (1985). Search asymmetry: A diagnostic for preattentive processing of separable features. Journal of Experimental Psychology: General, 114, 285-310

VoGels, R., \& ORBAN, G. A. (1987). Illusory contour orientation discrimination. Vision Research, 27, 453-467.

von der Heydt, R., \& Peterhans, E. (1989). Mechanisms of contour perception in monkey visual cortex: I. Lines of pattern discontinuity. Journal of Neuroscience, 9, 1731-1748.

von der Heydt, R., Peterhans, E., Baumgartner, G. (1984). Illusory contours and cortical neuron responses. Science, 224, 1260-1262.

Wallach, H., Slaughter, V. (1988). The role of memory in perceiving subjective contours. Perception \& Psychophysics, 43, 101-106.

Warm, J. S., Dember, W. N., Padich, R. A., Beckner, J., \& JONES, S. (1987). The role of illumination level in the strength of subjective contours. In S. Petry \& G. E. Meyer (Eds.), The perception of illusory contours (pp. 176-182). New York: Springer-Verlag.

\section{NOTES}

1. This interference may be of either sort outlined in the introduction: the circles increase interitem similarity and the display is originally organized as a homogeneous field of blobs, or the circles may provide luminance competition.

2. We have not included the block factor in the current analysis. Instead, the effect of blocks (i.e., practice) has been dealt with separately in the Appendix.

3. Kennedy (1988) has made this point convincingly, although informally. Following Grossberg and Mingolla (1985) - who argued that line terminations in gratings that induce subjective contours actually have an orientation component that is orthogonal to the line itself and therefore aligned with the subjective contour-Kennedy showed that the apparent strength of the subjective contour was greatly diminished if the line terminations were tapered rather than abrupt, that is, eliminating the orientation information available at the termination. Stimuli of the sort used in the present paper remain somewhat of a conundrum for Grossberg and Mingolla's analysis because they have trivial width and therefore very little in the way of orientation at the termination.

4. Treisman and Gormican (1988) make use of this observation in a somewhat different way. Rather than emphasizing the contrast within the display, they emphasize the contrast between displays with a target present and those with the target absent. When a target has magnitude $a$, it will contrast more strongly with the target-absent displays (i.e., all elements having magnitude $b$ ) than vice versa.

\section{APPENDIX}

In visual-search experiments, of course, the way in which RTs depend on the number of nontarget distractors is thought to re- veal something about the manner in which the display elements are processed. However, the interpretation of RTs may be compromised by practice effects. In any psychophysical experiment, we may expect performance to improve with practice as the subject becomes more familiar with the task. Therefore, relatively long RTs might reflect the combination of slow performance during the early stages of testing and much faster performance during later stages, and the asymptotic level of performance might not be indicated in this average. All experiments in this paper involved a manipulation of blocks that permits us to track performance over time. The purpose of the present Appendix is to describe the change in search slopes over time for Experiments $1 \mathrm{~A}$ and 2. These data are treated as supplementary because they do not alter the force of the points made in the main body of the text.

In all conditions of Experiments 1A, 1B, and 2, the stimuli were presented in 10 blocks of 48 trials per block, with eight replications of the six display-size $\times$ response-type conditions. Thus, the search slopes for the target-present and target-absent conditions were computed within each block, and the resulting two-factor, within-subject design $(10$ blocks $\times$ two response types) was subjected to an ANOVA. Seven such analyses were performed, one for each of the three conditions of Experiment $1 \mathrm{~A}$, one for each of the two conditions of Experiment 1B, and one for each of the two conditions of Experiment 2. Although in certain analyses there were significant differences between the two response types, in no analysis was there found an interaction of trial type with block. Therefore, we will restrict our analyses to the main effects of blocks in the seven conditions.

The block effects for each of the seven conditions are summarized in the left and right panels of Figure A1, and the results of the statistical analyses are shown in Table A1. As shown in Table A1, the block effect was significant only for Conditions 2 and 3 of Experiment 1A. Thus, in most cases, performance was stable across blocks, showing no effects of practice. For Condition 3 of Experiment $1 \mathrm{~A}$, the effects of practice appear to be limited to the first 2 blocks. To further assess this effect, we used two sets of nonorthogonal comparisons. First, it was found that Blocks 1 and 2 showed significantly longer average search slopes than did Blocks $3-10[F(1,11)=13.14$, $p<.005]$. However, a similar comparison of the average search slopes of Blocks 3-6 versus Blocks 7-10 showed no such significant difference $[F(1,11)=0.924, p>.35]$, indicating that after an initial improvement search rates stabilized rather quickly. The mean reaction times over the last 8 blocks were $3.98 \mathrm{msec} /$ item for target-present trials and $7.05 \mathrm{msec} / \mathrm{item}$ for target-absent trials.

Similar comparisons for Condition 2 revealed that Blocks 1 and 2 involved significantly longer average search slopes than did Blocks 3-10 $[F(1,11)=12.33, p<.005]$. However, the average search slopes of Blocks 3-6 versus Blocks $7-10$ also showed a significant difference $[F(1,11)=13.86, p<.005]$, indicating that improvement continued throughout the experiment.

It might be argued that the significant block effect in Condition 3 of Experiment 1A militates against our position that subjective contours-at least those in the present study - are the products of reflexive operations early in the visual system. It might be argued instead that what was initially a serial, resourcedemanding, constructive process has become automated over the course of the experiment. There are two responses to this point. The first is that the putative "parallelization" of a serial, constructive process must have occurred very quickly-over the 
Experiment $1 \mathrm{~A}$

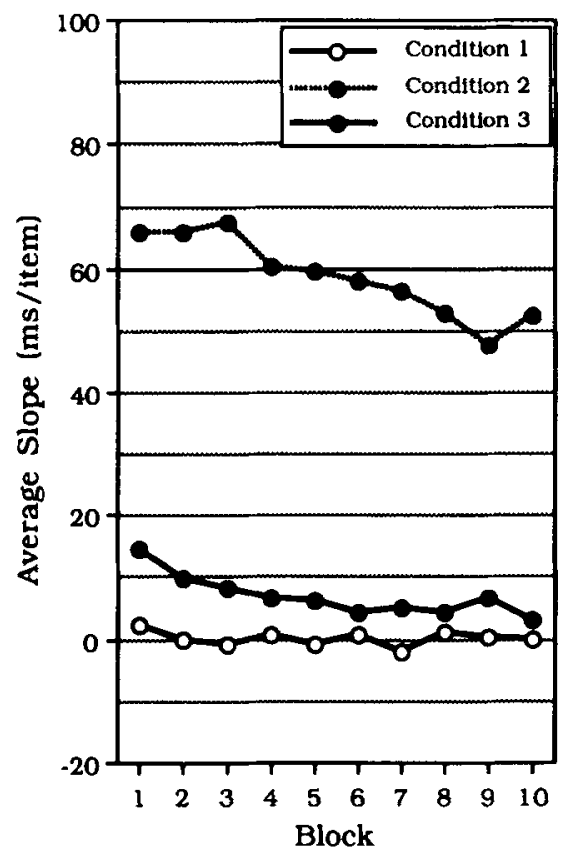

Experiments $1 \mathrm{~B}$ and 2

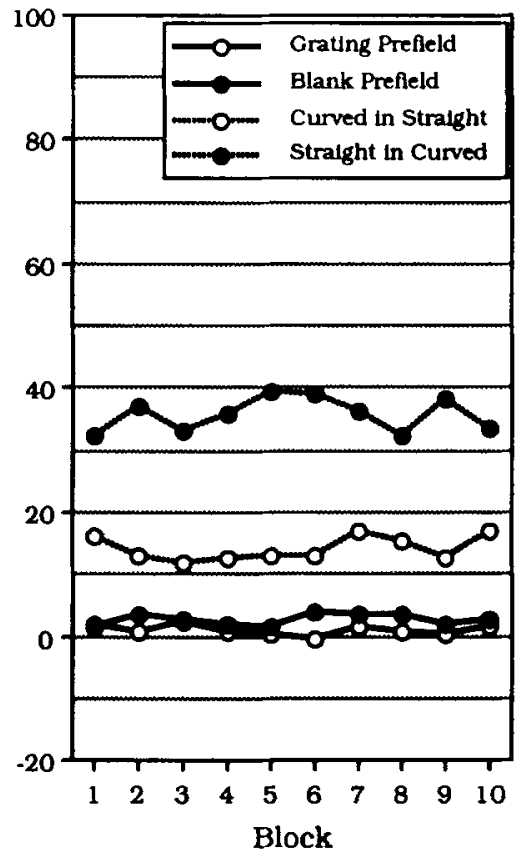

Figure A1. The left panel shows the change in reaction time slope (averaged over response type) as a function of blocks for the three conditions in Experiment 1A. The block effect is significant for Conditions 2 and 3. The right panel shows the same analysis for Experiments $1 B$ and 2 . In these cases, none of the block effects is significant.

course of two blocks of trials. This contrasts with the classic examples of learned automaticity found in the work of Schneider and Shiffrin (1977), in which automaticity emerged only after massive practice and so casts doubt on the proposition that these two sorts of improvement are of the same kind.

Second, the average search rates including the slower initial blocks are still very fast $(6.88 \mathrm{msec} / \mathrm{item})$ by the standards of the literature and even faster with these two blocks removed (5.52 msec/item). By appealing to the standards of the literature, we are not endorsing a Gallup-poll definition of parallelism, only pointing out that we are dealing with very fast search rates. More to the point, however, we have argued that subjective contours may be the products of very early processes but that access to such representations may be impaired by other features of the display. The results of Experiment $1 \mathrm{~A}$ are unquestionably consistent with this view. We did not claim that all noise in the display was eliminated by moving from Condition 2 to Condition 3 , only that it was reduced. There is most likely residual noise in the display that subjects must overcome. Over trials, subjects develop a model of the noise and factor it out as the experiment progresses. This seems a more parsimonious account of the data than one that posits the automatization of a resource-demanding, constructive process over the course of a few blocks.

Table A1

Summary of Block Analyses for Each of Seven Conditions Described in Experiments 1A, 1B, and 2

\begin{tabular}{cccc}
\hline Experiment & Condition & $F$ & $p$ \\
\hline IA & 1 & $(9,11)=0.08$ & $>.6$ \\
IA & 2 & $(9,11)=6.01$ & $<.001$ \\
1A & 3 & $(9,11)=2.97$ & $<.005$ \\
IB & Blank prefield & $(9,5)=0.54$ & $>.8$ \\
1B & Grating prefield & $(9,5)=0.58$ & $>.8$ \\
2 & Curved in straight & $(9,6)=0.59$ & $>.79$ \\
2 & Straight in curved & $(9,6)=0.45$ & $>.89$ \\
\hline
\end{tabular}

Note-The dependent measure was search slope averaged over trial type. 